Bundesgesundheitsbl 2013 ·56:439-446

DOI 10.1007/s00103-012-1625-x

Online publiziert: 17. Januar 2013

๑) Springer-Verlag Berlin Heidelberg 2013

\author{
A. Wienecke ${ }^{1,2} \cdot$ L. Knorpp ${ }^{1} \cdot$ K. Stegmüller ${ }^{1} \cdot A$. Kroke $^{1}$ \\ ${ }^{1}$ Hochschule Fulda \\ ${ }^{2}$ Zentrum für Krebsregisterdaten, Abteilung für Epidemiologie und \\ Gesundheitsberichterstattung, Robert Koch-Institut, Berlin
}

\title{
Präventionspotenziale für Darm- und Brustkrebs in Deutschland
}

\author{
Eine methodische Evaluation von \\ Schätzungen für die Risikofaktoren Alkohol \\ und Übergewicht
}

Die Zunahme der Zahl an Krebserkrankungen in den letzten Jahrzehnten stellt Politiker und medizinische Fachkräfte vor die Herausforderung, hier Handlungsbedarfe und -möglichkeiten sowie effektive Präventionsmaßnahmen zu identifizieren. Dazu ist eine Quantifizierung des Präventionspotenzials mit Blick auf verschiedene Risikofaktoren für Krebserkrankungen sinnvoll und nötig. Der folgende Beitrag verdeutlicht anhand exemplarischer Schätzungen der präventiven Potenziale bei den Risikofaktoren für Darm- und Brustkrebs in Deutschland die methodischen Schwierigkeiten einer solchen Quantifizierung.

\section{Hintergrund}

Die Zahl der Krebserkrankungen in Deutschland ist in den letzten Jahrzehnten kontinuierlich gestiegen [1]. Gründe für diese Entwicklung sind der demografische Wandel, die verbesserte Diagnostik sowie die zunehmende Prävalenz bestimmter Risikofaktoren, wie z. B. die Zunahme des Rauchens bei Frauen oder das vermehrte Auftreten von Adipositas. Nach Schätzungen des Robert Koch-Instituts (RKI) [1] ist die absolute Zahl der jährlichen Krebsneuerkrankungen in
Deutschland seit 1980 bei Frauen um 35\% und bei Männern um mehr als $80 \%$ gestiegen, während die altersstandardisierten Erkrankungsraten nur um 15\% bzw. 23\% zugenommen haben. Im Betrachtungszeitraum von 1999 bis 2008 zeigt sich für Männer eine konstante altersstandardisierte Erkrankungsrate, während diese für Frauen seit 2005 ansteigt. Trotz großer Unterschiede bei den Trends von Inzidenz und Mortalität einzelner Krebserkrankungen ist bei den häufigeren Tumorarten wie Darm- und Brustkrebs eine stetige Zunahme der Erkrankungszahlen zu verzeichnen. Dies lässt sich u. a. durch die Einführung entsprechender Screening-Programme für diese Erkrankungen erklären [1]. Projektionen zur Zahl der Krebsneuerkrankungen bis zum Jahr 2050 gehen von einem Anstieg um $26 \%$ auf jährlich etwa 642.000 Erkrankungsfälle aus (Gesamtzahl 2008: ca. 470.000 [1]), wobei zwischen den einzelnen Tumorarten deutliche Unterschiede zu erwarten sind [2].

Da viele Risikofaktoren wie „ungesunde“ Ernährung, körperliche Inaktivität, Tabak- und Alkoholkonsum vermieden werden können, werden Krebserkrankungen zu einem gewissen Anteil als verhinderbar angesehen $[3,4]$. Die- sen Risikofaktoren kommt daher in der Krebsprävention besondere Bedeutung zu. Schätzungen zufolge kann weltweit etwa ein Drittel der Krebserkrankungen durch den Verzicht auf Tabak und Alkohol, eine gesunde ausgewogene Ernährung und körperliche Aktivität verhindert werden $[3,4,5]$. Eine gesundheitsförderliche Lebensweise bietet somit ein erhebliches Präventionspotenzial. Der World Cancer Research Fund (WCRF) hat im Jahr 2009 Schätzungen zu eben diesem Präventionspotenzial für ausgewählte Länder veröffentlicht [4]. Diese sollen nicht nur das gesundheitspolitische und präventive Potenzial verdeutlichen, sondern auch zur Identifikation, Planung und Durchführung effektiver Public-Health-Interventionen beitragen $[6,7]$. In Ländern mit hohem Einkommensstatus wie den USA und Großbritannien könnten diesen Schätzungen zufolge bis zu 25\% aller Krebserkrankungen durch eine gesunde Ernährung und körperliche Aktivität vermieden werden [4].

Da Schätzungen zum Präventionspotenzial bei Krebserkrankungen aufgrund landesspezifischer Inzidenzraten und Risikofaktorprävalenzen stets populationsspezifisch sind [8], ist es sinnvoll, gesonderte Berechnungen für Deutsch- 


\section{Originalien und Übersichten}

Tab. 1 Überblick über verwendete Studien, relative Risiken und Expositionskategorien

\begin{tabular}{|c|c|c|c|c|c|c|c|c|}
\hline & \multicolumn{4}{|l|}{ Darmkrebs } & \multicolumn{4}{|l|}{ Brustkrebs } \\
\hline & Wei et al. $[10]^{a}$ & $\begin{array}{l}\text { Ferrari et al. } \\
{[11]^{\text {b }}}\end{array}$ & $\begin{array}{l}\text { Engeland et } \\
\text { al. [12] }{ }^{\mathrm{a}}\end{array}$ & $\begin{array}{l}\text { Pischon et al. } \\
{[13]^{b}}\end{array}$ & $\begin{array}{l}\text { Willett et } \\
\text { al. }[14]^{a}\end{array}$ & $\begin{array}{l}\text { Tjonneland } \\
\text { et al. }[15]^{\mathrm{b}}\end{array}$ & $\begin{array}{l}\text { van den } \\
\text { Brandt et al. } \\
{[16]^{\mathrm{a}}}\end{array}$ & $\begin{array}{l}\text { Lahmann et } \\
\text { al. [17] }{ }^{\text {b }}\end{array}$ \\
\hline Exposition & Alkohol (g/Tag) & $\begin{array}{l}\text { Alkohol (g/ } \\
\text { Tag) }\end{array}$ & BMI $\left(\mathrm{kg} / \mathrm{m}^{2}\right)$ & BMI $\left(\mathrm{kg} / \mathrm{m}^{2}\right)$ & $\begin{array}{l}\text { Alkohol (g/ } \\
\text { Tag) }\end{array}$ & $\begin{array}{l}\text { Alkohol (g/ } \\
\text { Tag) }\end{array}$ & BMI $\left(\mathrm{kg} / \mathrm{m}^{2}\right)$ & BMI $\left(\mathrm{kg} / \mathrm{m}^{2}\right)$ \\
\hline Endpunkt & Kolonkrebs & $\begin{array}{l}\text { Kolorektal- } \\
\text { krebs }\end{array}$ & $\begin{array}{l}\text { Kolorektal- } \\
\text { krebs }\end{array}$ & Kolonkrebs & Brustkrebs & Brustkrebs & $\begin{array}{l}\text { Postmeno- } \\
\text { pausaler } \\
\text { Brustkrebs }\end{array}$ & $\begin{array}{l}\text { Postmeno- } \\
\text { pausaler } \\
\text { Brustkrebs }\end{array}$ \\
\hline $\begin{array}{l}\text { Studiende- } \\
\text { sign }\end{array}$ & $\begin{array}{l}\text { Kohortenstudie, } \\
\text { USA }\end{array}$ & $\begin{array}{l}\text { Kohorten- } \\
\text { studie, Europa } \\
\text { (EPIC) }\end{array}$ & $\begin{array}{l}\text { Kohorten- } \\
\text { studie, Nor- } \\
\text { wegen }\end{array}$ & $\begin{array}{l}\text { Kohorten- } \\
\text { studie, Europa } \\
\text { (EPIC) }\end{array}$ & $\begin{array}{l}\text { Kohorten- } \\
\text { studie, USA }\end{array}$ & $\begin{array}{l}\text { Kohortenstu- } \\
\text { die, Europa } \\
\text { (EPIC) }\end{array}$ & $\begin{array}{l}\text { Analyse von } \\
7 \text { Kohorten- } \\
\text { studien, } \\
\text { Europa, USA }\end{array}$ & $\begin{array}{l}\text { Kohortenstu- } \\
\text { die, Europa } \\
\text { (EPIC) }\end{array}$ \\
\hline $\begin{array}{l}\text { Studienpopu- } \\
\text { lation }\end{array}$ & $\begin{array}{l}\text { Teilnehmer der } \\
\text { Nurses Health } \\
\text { Study ( } 87.733 \text { Per- } \\
\text { sonen, } 30 \text { bis } \\
55 \text { Jahre) und der } \\
\text { Health Professional } \\
\text { Follow-up Study } \\
\text { (46.632 Personen, } \\
40 \text { bis } 75 \text { Jahre) }\end{array}$ & $\begin{array}{l}\text { 478.732 Per- } \\
\text { sonen } \\
\text { ( } 142.851 \text { Män- } \\
\text { ner, } \\
335.881 \text { Frau- } \\
\text { en) } 35 \text { bis } \\
70 \text { Jahre }\end{array}$ & $\begin{array}{l}2 \text { Mio. Frauen } \\
\text { und Männer, } \\
20 \text { bis } 74 \text { Jah- } \\
\text { re }\end{array}$ & $\begin{array}{l}\text { 368.277 Per- } \\
\text { sonen } \\
\text { ( } 129.731 \text { Män- } \\
\text { ner, } \\
238.546 \text { Frau- } \\
\text { en) } 35 \text { bis } \\
70 \text { Jahre }\end{array}$ & $\begin{array}{l}89.538 \text { Frau- } \\
\text { en, } 34 \text { bis } \\
59 \text { Jahre, } \\
\text { Kranken- } \\
\text { schwestern }\end{array}$ & $\begin{array}{l}274.688 \text { Frau- } \\
\text { en, } 35 \text { bis } \\
70 \text { Jahre }\end{array}$ & $\begin{array}{l}337.819 \text { Frau- } \\
\text { en, } 30 \text { bis } \\
90 \text { Jahre }\end{array}$ & $\begin{array}{l}176.886 \text { Frau- } \\
\text { en, } 18 \text { bis } \\
80 \text { Jahre }\end{array}$ \\
\hline $\begin{array}{l}\text { Datenerhe- } \\
\text { bung }\end{array}$ & $\begin{array}{l}\mathrm{FFQ} \text { - semiquan- } \\
\text { titativ }\end{array}$ & $\begin{array}{l}\text { Validierter Fra- } \\
\text { gebogen }\end{array}$ & $\begin{array}{l}\text { Standardisier- } \\
\text { te Messung }\end{array}$ & $\begin{array}{l}\text { Standardisier- } \\
\text { te Messung }\end{array}$ & $\begin{array}{l}\text { FFQ - semi- } \\
\text { quantitativ }\end{array}$ & $\begin{array}{l}\text { Validierter } \\
\text { Fragebogen }\end{array}$ & $\begin{array}{l}\text { FFQ, Fra- } \\
\text { gebogen } \\
\text { (Selbstanga- } \\
\text { ben) }\end{array}$ & $\begin{array}{l}\text { Standardi- } \\
\text { sierte Mes- } \\
\text { sung }\end{array}$ \\
\hline $\begin{array}{l}\text { Expositions- } \\
\text { kategorien } \\
\text { (WCRF) }\end{array}$ & $0,0-20,>20$ & $0,0-15,>15$ & $\begin{array}{l}<25,25-30 \\
>30\end{array}$ & $\begin{array}{l}<25,25-30 \\
>30\end{array}$ & $0,0-15,>15$ & $0,0-10,>10$ & $\begin{array}{l}<21,21-25 \\
>25\end{array}$ & $\begin{array}{l}<21,21-25 \\
>25\end{array}$ \\
\hline RR (Männer) & 1,$00 ; 1,27 ; 1,55$ & 1,$00 ; 1,05 ; 1,26$ & $\begin{array}{l}1,00 ; 1,15 ; \\
1,40\end{array}$ & $\begin{array}{l}1,00 ; 1,00 ; \\
1,41\end{array}$ & - & - & - & - \\
\hline RR (Frauen) & 1,$00 ; 0,99 ; 1,14$ & 1,$00 ; 1,05 ; 1,26$ & $\begin{array}{l}1,00 ; 1,02 ; \\
1,06\end{array}$ & $\begin{array}{l}1,00 ; 1,16 ; \\
1,07\end{array}$ & 1,$00 ; 1,3 ; 1,6$ & $\begin{array}{l}1,00 ; 0,97 ; \\
1,07\end{array}$ & $\begin{array}{l}1,00 ; 1,15 ; \\
1,26\end{array}$ & $\begin{array}{l}1,00 ; 1,35 ; \\
1,38\end{array}$ \\
\hline $\begin{array}{l}\text { Alternative } \\
\text { Expositions- } \\
\text { kategorien }\end{array}$ & $<10,10-20,>20$ & $<5,5-15,>15$ & $\begin{array}{l}\text { Nicht nötig, } \\
\text { da bereits } \\
\text { praxisrelevan- }\end{array}$ & $\begin{array}{l}\text { Nicht nötig, } \\
\text { da bereits pra- } \\
\text { xisrelevante }\end{array}$ & $\begin{array}{l}<5,5-15 \\
>15\end{array}$ & $<5,5-10,>10$ & $\begin{array}{l}<25,25-30 \\
>30\end{array}$ & $\begin{array}{l}<25,25-30 \\
>30\end{array}$ \\
\hline RR (Männer) & 1,$00 ; 1,27 ; 1,55$ & 1,$00 ; 1,05 ; 1,26$ & & Referenzkate- & - & - & - & - \\
\hline RR (Frauen) & 1,$00 ; 0,99 ; 1,14$ & 1,$00 ; 1,05 ; 1,26$ & Kategorle & & 1,$00 ; 1,3 ; 1,6$ & $\begin{array}{l}1,00 ; 0,97 \\
1,07\end{array}$ & $\begin{array}{l}1,00 ; 1,43 \\
1,27\end{array}$ & $\begin{array}{l}1,00 ; 1,30 \\
1,31\end{array}$ \\
\hline
\end{tabular}

land mittels nationaler Daten vorzunehmen.

Ziel der vorliegenden Arbeit war es, beispielhaft für Darm- und Brustkrebserkrankungen in Deutschland Schätzungen zum Präventionspotenzial mit Blick auf 2 ausgewählte evidenzbasierte Risikofaktoren [Alkoholkonsum und Übergewicht (hier: BMI)] vorzunehmen und anhand des Einsatzes unterschiedlicher Berechnungsparameter zu zeigen, welche Faktoren das Ergebnis solcher Schätzungen beeinflussen und wie stark sich die Ergebnisse in Abhängigkeit der gewählten Parameter voneinander unterscheiden.

\section{Material und Methoden}

Für die exemplarische Berechnung wurden die evidenzbasierten Risikofaktoren Body Mass Index (BMI) und Alkoholkonsum ausgewählt. Systematische Reviews des WCRF zeigen für beide Faktoren überzeugende Evidenz dafür, dass sie das Darm- bzw. Brustkrebsrisiko erhöhen [3]. Diese beiden Krebsarten sind auch aufgrund der Häufigkeit ihres Auftretens von besonderer Bedeutung für Deutschland [1].

In einem ersten Schritt erfolgte die Berechnung der populationsattributablen Fraktionen (PAF) für Deutschland mittels der vom WCRF angewandten Me- thodik, um eine Vergleichbarkeit der Ergebnisse mit den WCRF-Schätzungen zu ermöglichen. In einem zweiten Schritt wurden die Expositionskategorien und Risikoschätzer variiert, um die Auswirkungen einer Veränderung von Cut-offWerten (Grenzwerten) und relativen Risiken auf die Ergebnisse der Schätzung zu untersuchen. Im Allgemeinen sind für die PAF-Berechnungen die Prävalenzen in den für die jeweiligen Risikofaktoren festgelegten Expositionskategorien sowie die relativen Risiken (RR) zum $\mathrm{Zu}$ sammenhang zwischen den Risikofaktoren und den gewählten Krebsarten in den entsprechenden Expositionskategorien erforderlich. 


\section{Studienpopulation/ Risikofaktorenprävalenz}

Die verwendeten Daten zum Alkoholkonsum und BMI der deutschen Bevölkerung stammen aus der Nationalen Verzehrsstudie II (NVS II; [9]). Die NVS II basiert auf einer repräsentativen Stichprobe ( $n=19.329)$ der deutschen Bevölkerung im Alter zwischen 14 und 80 Jahren, in der von Januar 2006 bis Januar 2007 Daten zur Lebensmittel- und Nährstoffzufuhr sowie zur Anthropometrie (Körpermaße) mittels standardisierter und validierter Erhebungs- und Messmethoden erfasst wurden. Die genauen Methoden der Datenerhebung können im Ergebnisteil I der NVS II nachgelesen werden [9].

Die für die PAF-Berechnungen benötigten Prävalenzschätzungen in den unterschiedlichen Expositionskategorien wurden auf Basis der Scientific Use Files der NVS II ermittelt [9]. Hierzu wurden die Daten zum Alkoholkonsum und BMI in je 3 Kategorien überführt, wobei die Grenzwerte der jeweiligen Expositionskategorien gemäß der WCRF-Methodik bzw. die aus den ausgewählten Kohortenstudien verwendeten Grenzwerte übernommen wurden (s. Berechnungen der PAF).

\section{Relative Risiken}

Die relativen Risiken zum Zusammenhang zwischen Alkoholkonsum/Übergewicht und Darm- bzw. Brustkrebs stammen zum einen aus den Kohortenstudien, die auch der WCRF für seine Berechnungen zum präventiven Potenzial herangezogen hat [4], um eine Vergleichbarkeit der Ergebnisse mit denen des WCRF zu ermöglichen. Zum anderen wurden Risikoschätzer aus anderen ausgewählten Kohortenstudien eingesetzt, um die Auswirkungen unterschiedlicher Risikoschätzer und Expositionskategorien auf die Ergebnisse der Schätzungen zu verdeutlichen. Dazu wurden ausnahmslos Studien der European Investigation into Cancer and Nutrition (EPIC) herangezogen (- Tab. 1), da hier Daten aus Deutschland in die Berechnungen der RR eingeflossen sind und so die Ergebnisse der PAF-Schätzungen besser auf Deutschland übertragen werden können [8]. Die wesentlichen

Bundesgesundheitsbl 2013 · 56:439-446 DOI 10.1007/s00103-012-1625-x

(c) Springer-Verlag Berlin Heidelberg 2013

\section{A. Wienecke · L. Knorpp · K. Stegmüller · A. Kroke \\ Präventionspotenziale für Darm- und Brustkrebs in Deutschland. Eine methodische Evaluation von Schätzungen für die Risikofaktoren Alkohol und Übergewicht}

\section{Zusammenfassung}

Schätzungen zum präventiven Potenzial für die Risikofaktoren Alkohol und Übergewicht (bestimmt durch den BMI) bei Darm- und Brustkrebs wurden beispielhaft für Deutschland berechnet, um anschließend die Problematik der Schätzung von Präventionspotenzialen anhand von Vergleichsrechnungen zu diskutieren. Basierend auf Daten der Nationalen Verzehrsstudie II zum Alkoholkonsum und zur Anthropometrie (Körpermaße) der deutschen Bevölkerung wurden mittels verschiedener Risikoschätzer populationsattributable Fraktionen (PAF) berechnet. Die PAF beziffert den Anteil, um den sich das Auftreten einer Erkrankung verringern ließe, wenn der Krankheitsauslöser beseitigt oder reduziert würde. Hinsichtlich Darmkrebs ergab sich für Männer ein präventives Potenzial beim BMI von 7-13\% und beim Alkoholkonsum von $10-25 \%$. Das präventive Potenzial eines niedrigen BMI bei postmenopausalem Brustkrebs lag zwischen 13 und 23\%. Die Vergleichsrechnungen zeigten, dass die Ergebnisse der Schätzungen stark von den gewählten Daten (Risikoschätzern, Referenzkategorien) abhingen und sich um bis zu 50\% voneinander unterschieden. Um zukünftig validere Schätzungen zum Präventionspotenzial zu ermöglichen, sollte die Datenauswahl evidenzbasiert erfolgen und, wenn möglich, auf Risikoschätzer aus Metaanalysen zurückgegriffen werden.

Schlüsselwörter

Krebsprävention · Präventionspotenzial · Alkoholkonsum · Übergewicht · Deutschland

\section{Preventability estimates for colorectal and breast cancer in Germany. A methodological evaluation of the risk factors alcohol and overweight}

\section{Abstract}

Population attributable fractions (PAFs) for the risk factors alcohol intake and overweight in the German population were calculated to estimate the preventability of colorectal and breast cancer attributable to these risk factors. Estimates were based on national alcohol consumption and overweight prevalence data in the German population. Comparative analyses were used to evaluate the variation of PAF estimates according to changes in the calculation parameters. PAFs quantify the preventive potential that could result from removing or reducing the risk factor exposure, respectively. Postmenopausal breast cancer was estimated to be preventable by $13-23 \%$ if the population were nor- mal weight. Among German men, $10-25 \%$ of colon cancers were attributable to alcohol consumption and $7-13 \%$ of colorectal cancers to overweight. The comparative analyses demonstrated that preventability estimates vary considerably with the chosen data (risk estimates, reference categories) for PAF calculation and can differ by up to $50 \%$. Thus, data selection should be evidence based, for example, based on meta-analyses, in order to increase the validity of preventability estimates.

Keywords

Cancer prevention - Preventability estimates . Alcohol consumption · Overweight · Germany
Merkmale der Studien sowie die verwendeten Expositionskategorien und relativen Risiken sind in $\bullet$ Tab. 1 aufgeführt.

\section{Berechnungen der PAF}

Die Schätzungen zum präventiven Potenzial erfolgten geschlechtsspezifisch und mittels der Formel zur Berechnung der populationsattributablen Fraktionen nach dem Konzept von Levin [18]. Die populationsattributable Fraktion gibt den Anteil an, um den die Krankheitsinzidenz in einer Bevölkerung reduziert werden kann, wenn die ursächliche Exposition eliminiert oder auf das niedrigste mögliche Expositionsniveau reduziert wird [6]. Bei dieser Methode wird die Expositionskategorie mit dem niedrigsten Risiko als Referenzkategorie gewählt. Für risikoerhöhen- 
Tab. 2 Charakteristika der in die Berechnung einbezogenen Studienpopulation.

[Nach Daten der Nationalen Verzehrsstudie II (NVS II, Scientific Use Files, [9])]

\begin{tabular}{|llll}
\hline & Männer & Frauen & \\
\cline { 3 - 3 } & & Alle Altersgruppen & Postmenopausal \\
\hline Anzahl (n) & 8932 & 10.406 & 3562 \\
\hline Alter in Jahren & & $63(8,1)$ \\
\hline Median (SD) & $47(18,3)$ & $46(17,5)$ & \\
\hline Alkoholkonsum (g/Tag) & & $2(9,0)$ \\
\hline Median (SD) & $9(18,3)$ & $2(8,4)$ & $27,5(27,3-27,7)$ \\
\hline BMI (kg/m $)$ & & - \\
\hline MW (95\%-KI) & $26,6(26,5-26,7)$ & $25,7(25,6-25,9)$ & \\
\hline Anteil Raucher (\%) & 30 & 24 & \\
\hline SD Standardabweichung, MW Mittelwert, KI Konfidenzintervall. & \\
\hline
\end{tabular}

Tab. 3 PAF-Schätzungen für Deutschland; Vergleichende Darstellung der berechneten Potenziale mit den vom World Cancer Research Fund (WCRF) ermittelten Schätzungen für die USA und UK. (Nach [4])

\begin{tabular}{|c|c|c|c|c|}
\hline & \multicolumn{4}{|l|}{ PAF $\%$} \\
\hline & \multicolumn{2}{|l|}{ Darmkrebs } & \multicolumn{2}{|l|}{ Brustkrebs } \\
\hline & $\mathrm{BMI}(<25)$ & Alkohol $(0 \mathrm{~g} / \mathrm{Tag})^{\mathrm{a}}$ & $\operatorname{BMI}(<21)^{b}$ & Alkohol (0 g/Tag) \\
\hline \multicolumn{5}{|c|}{ Deutschland } \\
\hline Männer & 13 & 25 & - & - \\
\hline Frauen & 2 & $-1,6$ & 17 & 24 \\
\hline \multicolumn{5}{|l|}{ USA } \\
\hline Männer & 16 & \multirow[t]{2}{*}{$5^{c}$} & - & - \\
\hline Frauen & 3 & & 17 & 11 \\
\hline \multicolumn{5}{|l|}{ UK } \\
\hline Männer & 14 & \multirow[t]{2}{*}{$7^{c}$} & - & - \\
\hline Frauen & 2 & & 16 & 22 \\
\hline
\end{tabular}

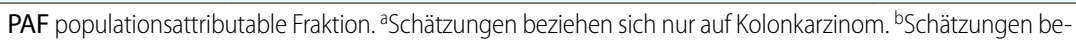
ziehen sich nur auf postmenopausalen Brustkrebs. PPAF nicht geschlechtsspezifisch.

de Expositionen wie Alkohol und Übergewicht entspricht dies dem niedrigsten $\mathrm{Zu}$ fuhr- bzw. BMI-Level.

$$
\begin{aligned}
& {[P F 1 \cdot(R R 1-1)]+[P F 2 .} \\
& (R R 2-1)]+[P F 3 . \\
P A F \%= & \frac{(R R 3-1)]}{[P F 1 \cdot(R R 1-1)]+[P F 2 \cdot} \cdot 100 \\
& (R R 2-1)]+[P F 3 . \\
& (R R 3-1)]+1
\end{aligned}
$$

wobei:

- PF1 = Bevölkerungsanteil in der Expositionskategorie mit niedrigem $\mathrm{Ri}$ siko,

- RR1 = Referenzkategorie (relatives Risiko von 1,0 für die niedrigste Expositionskategorie),

- PF2 = Bevölkerungsanteil in der Expositionskategorie mit mittlerem Risiko,
- RR2 = relatives Risiko der Expositionskategorie mit mittlerem Risiko,

- PF3 = Bevölkerungsanteil in der Expositionskategorie mit hohem Risiko,

- RR3 = relatives Risiko der Expositionskategorie mit hohem Risiko.

Da die Kategorisierung der Risikoexposition in den für die Berechnungen herangezogenen Kohortenstudien zum Teil mit mehr als 3 Expositionskategorien erfolgte, mussten zunächst die relativen Risiken für die 3 Expositionskategorien niedriges (RR1), mittleres (RR2) und hohes Risiko (RR3) ermittelt werden. Hier wurde auf die Vorgabe zu den Expositionskategorien und RR aus dem WCRF-Report Bezug genommen. Entsprechend wurden als Referenzkategorien bei den Berechnungen für den Risikofaktor Übergewicht (BMI: Körpergewicht geteilt durch Körpergröße $\left(\mathrm{kg} / \mathrm{m}^{2}\right)$ ) bei Brustkrebs ein BMI $<21$ und bei Darmkrebs ein BMI $<25$ festgelegt. Für den Risikofaktor Alkoholkonsum wurde als Referenzkategorie eine Exposition von $0 \mathrm{~g} / \mathrm{Tag}$ verwendet. Diese den Referenzkategorien des WCRFReports entsprechenden Expositionskategorien [4] repräsentieren das theoretische minimale Risiko [19]. Da es sich beim theoretisch minimalen Risikos um in der Praxis schwer umsetzbare $(0 \mathrm{~g} \mathrm{Al}$ kohol/Tag; [20]) bzw. möglicherweise in anderer Hinsicht nicht gesundheitsförderliche (BMI <21; [21]) Präventionsziele handelt, wurden PAF-Schätzungen unter Verwendung alternativer Referenzkategorien vorgenommen. Im Falle des Alkoholkonsums wurde die Referenzkategorie mit $<10 \mathrm{~g} /$ Tag für Darmkrebs und $<5 \mathrm{~g} /$ Tag für Brustkrebs definiert. Hinsichtlich des BMI wurde jeweils ein Referenzwert von $<25$ festgelegt.

Schließlich wurden weitere relative $\mathrm{Ri}$ siken aus anderen Kohortenstudien verwendet, um den starken Einfluss des Risikoschätzers auf die PAF-Schätzung zu verdeutlichen. Die Referenzkategorien entsprechen denen aus dem WCRF-Report bzw. den alternativ definierten Referenzkategorien (s. oben). Sofern zur Ermittlung der 3 Expositionskategorien und der entsprechenden relativen Risiken mehrere originale Kategorien einer Kohortenstudie zu einer Kategorie zusammengefasst werden mussten, wurde der Wert der höchsten Kategorie als RR für die Berechnungen gewählt. Beim Zusammenfassen von mehreren Expositionskategorien zu einer Kategorie mit nach oben hin offenem Grenzwert (beispielsweise BMIKategorien 30-31, 31-33, > 33 zu Kategorie $>30$ ) wurde der Wert der niedrigeren Expositionskategorie (30-31) gewählt.

Da bezüglich des BMI nur für den postmenopausalen Brustkrebs eine überzeugende risikoerhöhende Evidenz vorliegt, wurden in die Berechnungen zum BMI bei Brustkrebs nur Frauen ab einem Alter von 50 Jahren einbezogen, da dieses Alter im Allgemeinen als postmenopausal angesehen werden kann [22].

\section{Ergebnisse}

Zur Berechnungen des Präventionspotenzials bei den Darm- und Brustkrebsrisikofaktoren Alkohol und Übergewicht lagen insgesamt Daten von 10.406 Frauen 
Tab. 4 PAF-Schätzungen für Deutschland, basierend auf unterschiedlichen

Referenzkategorien ${ }^{\mathrm{a}}$

\begin{tabular}{|c|c|c|c|c|c|c|}
\hline & \multicolumn{6}{|l|}{ PAF $\%$} \\
\hline & \multicolumn{2}{|c|}{ Darmkrebs ${ }^{b}$} & \multicolumn{4}{|c|}{ Brustkrebs } \\
\hline & \multicolumn{2}{|l|}{ Alkohol } & \multicolumn{2}{|l|}{ BMIC } & \multicolumn{2}{|l|}{ Alkohol } \\
\hline & $0 \mathrm{~g} / \mathrm{Tag}$ & $<10 \mathrm{~g} / \mathrm{Tag}$ & $<21$ & $<25$ & $0 \mathrm{~g} / \mathrm{Tag}$ & $<5 \mathrm{~g} / \mathrm{Tag}$ \\
\hline Männer & 25 & 17 & - & - & - & - \\
\hline Frauen & $-1,6$ & 1 & 17 & 13 & 24 & 11 \\
\hline
\end{tabular}

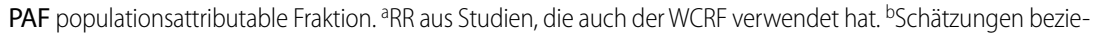
hen sich nur auf Kolonkarzinom. SSchätzungen beziehen sich nur auf postmenopausalen Brustkrebs.

Tab. 5 PAF-Schätzungen für Deutschland unter Verwendung alternativer relativer Risiken aus Datenanalysen der EPIC-Kohorte

\begin{tabular}{|c|c|c|c|c|c|c|c|}
\hline & \multicolumn{7}{|c|}{ PAF \% } \\
\hline & \multicolumn{3}{|c|}{ Darmkrebs } & \multicolumn{4}{|c|}{ Brustkrebs } \\
\hline & \multirow{2}{*}{$\begin{array}{l}\text { BMI }^{\mathrm{a}} \\
<25\end{array}$} & \multicolumn{2}{|l|}{ Alkohol } & \multicolumn{2}{|c|}{$\mathrm{BM} \mathrm{I}^{\mathrm{b}}$} & \multicolumn{2}{|l|}{ Alkohol } \\
\hline & & $0 \mathrm{~g} / \mathrm{Tag}$ & $<5 \mathrm{~g} / \mathrm{Tag}$ & $<21$ & $<25$ & $0 \mathrm{~g} / \mathrm{Tag}$ & $<5 \mathrm{~g} / \mathrm{Tag}$ \\
\hline Männer & 7 & 11 & 10 & - & - & - & - \\
\hline Frauen & 6 & 6 & 4 & 23 & 16 & $-1,1$ & 1 \\
\hline
\end{tabular}

PAF populationsattributable Fraktion, EPIC European Prospective Investigation into Cancer and Nutrition.

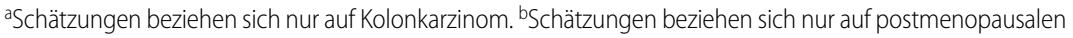
Brustkrebs.

und 8932 Männern aus der NVS II vor. In die Berechnungen zum Einfluss des BMI bei Darmkrebs sind Daten von 6753 Männern und 7796 Frauen eingeflossen, in die Berechnungen zum Einfluss des Alkoholkonsums bei Darm- und Brustkrebs Daten von 7093 Männern und 8278 Frauen. Für die Berechnungen des präventiven Potenzials beim Risikofaktor BMI für postmenopausalen Brustkrebs lagen Daten von 3562 Frauen $>50$ Jahren vor. Die geschlechtsspezifischen Verteilungen der Risikofaktoren sind in $\bullet$ Tab. 2 dargestellt.

Die Berechnungen zum präventiven Potenzial gemäß WCRF-Methodik ergaben für den postmenopausalen Brustkrebs (Frauen $>50$ Jahre) eine Verringerung von $17 \%$ bei einer Reduktion des BMI auf <21. Bei völligem Verzicht auf Alkohol zeigte sich bei Frauen aller Altersstufen eine Verringerung um 24\%. Das Präventionspotenzial einer Reduzierung des Alkoholverzehrs oder des BMI zur Vermeidung von Darmkrebs fiel dagegen wesentlich geringer aus. Demnach ließe sich die Zahl der Darmkrebsfälle bei Frauen bei vollständigem Alkoholverzicht gar nicht und bei einer Reduzierung des BMI auf $<25$ lediglich um $2 \%$ verringern. Im Vergleich dazu lag das präventive Potenzial bezüglich Darmkrebs bei Män- nern für beide Risikofaktoren deutlich höher. Hier ergaben die Berechnungen nach WCRF-Methodik ein Präventionspotenzial von $13 \%$ bei Reduktion des BMI auf $<25$ und von $25 \%$ bei einer Verringerung des Alkoholkonsums auf $0 \mathrm{~g} /$ Tag. Insgesamt entsprechen diese Schätzungen in ihrer Größenordnung den Berechnungen des WCRF für die USA und Großbritannien (UK; • Tab. 3).

Um bei der Schätzung des Präventionspotenzials die Erreichbarkeit der Risikofaktormodifikation zu berücksichtigen, wurden die PAF-Berechnungen unter Verwendung alternativer Referenzkategorien wiederholt. Hierbei zeigte sich im Vergleich zu den vorherigen Schätzungen für postmenopausalen Brustkrebs bei einer Reduktion des BMI auf $<25$ ein Präventionspotenzial von $13 \%$ (vs. $17 \%$ bei einem BMI <21) und ein Potenzial von $11 \%$ bei einer Reduzierung des Alkoholkonsums auf $<5 \mathrm{~g} / \mathrm{Tag}$ (vs. $24 \%$ bei $0 \mathrm{~g} /$ Tag). Für Darmkrebs bei Frauen ergab sich bei einer Referenzkategorie für $<10 \mathrm{~g}$ Alkohol/Tag ein präventiver Anteil von $1 \%$, bei Männern von $17 \%$ (vs. $-1,6 \%$ bzw. 25\% bei $0 \mathrm{~g} / \mathrm{Tag}$ ) (• Tab. 4).

Um den Einfluss der verwendeten $\mathrm{Ri}$ sikoschätzer auf die Größe des geschätzten Präventionspotenzials darzustellen, wurden die PAF-Berechnungen schließ- lich erneut wiederholt, indem andere relative Risiken eingesetzt wurden. Hierzu wurden sowohl die WCRF-Referenzkategorien als auch die alternativen $\mathrm{Re}$ ferenzkategorien für die Neuberechnung genutzt (• Tab.5). Durch Verwendung anderer RR fiel das Präventionspotenzial mit Blick auf Darmkrebs bei vollständigem Verzicht auf Alkohol bei Männern um 14\%-Punkte geringer und bei Frauen um $8 \%$-Punkte größer aus. Bei einer Reduzierung des Alkoholkonsums auf $<5$ g/ Tag ergaben die alternativen Risikoschätzer ein präventives Potenzial von $10 \%$ bei Männern und von $4 \%$ bei Frauen. Auch die PAF-Ergebnisse für Brustkrebs und Alkoholkonsum unterschieden sich erheblich. So errechnete sich bei Verwendung der RR aus der Studie von Willett et al. [14] ein präventives Potenzial von $11 \%$ (Alkoholkonsum <5 g/Tag) und 24\% (Alkoholkonsum $0 \mathrm{~g} / \mathrm{Tag}$ ), während sich bei Verwendung der RR aus der Studie von Tjonneland et al. [15] lediglich ein Präventionspotenzial von maximal $1 \%$ ergab. Die Ergebnisse zu Brustkrebs und BMI unterschieden sich hingegen weniger stark (vgl.

- Tab. 4 und 5).

\section{Diskussion}

In der vorliegenden Arbeit wurden Schätzungen zum präventiven Potenzial bei den Darm- und Brustkrebsrisikofaktoren Alkoholkonsum und Übergewicht in Deutschland berechnet und mit den Schätzungen des WCRF für die USA und Großbritannien [3] verglichen. Dabei wurde untersucht, wie stark sich die Schätzungen bei Verwendung unterschiedlicher Berechnungsparameter (Referenzkategorien, Risikoschätzer) verändern. Während die PAF-Schätzungen für Deutschland bei Verwendung der WCRFBerechnungsparameter weitgehend mit den Schätzungen für die USA und Großbritannien übereinstimmen (• Tab. 3), variierte das berechnete präventive Potenzial bei Verwendung alternativer Referenzkategorien und relativer Risiken zum Teil erheblich (• Tab. 4 und 5). So ergab sich für Darmkrebs ein präventives Potenzial beim BMI von 7-13\% (Männer) bzw. 2-6\% (Frauen) und für den Alkoholkonsum von 10-25\% (Männer) bzw. von -1,6 bis $6 \%$ (Frauen). Am stärksten veränder- 
ten sich die Schätzungen für postmenopausalen Brustkrebs, die beim Risikofaktor Alkoholkonsum zwischen -1,1 und $24 \%$ und beim BMI zwischen 13 und 23\% schwankten.

\section{Vergleich mit anderen Studien}

Schätzungen zum Präventionspotenzial bei verschiedenen Umwelt- und Lebensstilfaktoren für spezifische Krebserkrankungen liegen unter anderem für die WHO-Regionen [23, 24, 25], Europa [26], die USA [27], Frankreich [28], Großbritannien [29] und Skandinavien [30] vor. Diese sind aufgrund von Unterschieden bei den verwendeten RR, Expositionskategorien und Risikoprävalenzen sowie von Variationen in der angewandten Methodik in ihren Ergebnissen nur bedingt miteinander vergleichbar. Für Deutschland wurden populationsspezifische Präventionspotenziale für Brust- und Darmkrebs unter anderem in 2 weiteren Studien ermittelt, wobei die Ergebnisse im Bereich der hier berechneten Schätzungen liegen. In einer Untersuchung zur alkoholattributablen Last für die Krebsinzidenz berechneten die Autoren ein präventives Potenzial für Darmkrebs von $16 \%$ (Männer) bzw. 6\% (Frauen) und für Brustkrebs von 7\% [31]. Für den Risikofaktor BMI schätzten Renehan et al. [26] das Präventionspotenzial in Deutschland hinsichtlich Darmkrebses bei Männern auf 14\% und bei Frauen auf 3\%. Lediglich für den postmenopausalen Brustkrebs fiel das geschätzte Potenzial beim BMI mit knapp 6\% konservativer aus als in der vorliegenden Schätzungen. Allerdings beruhen die Berechnungen von Renehan et al. auf der Annahme einer Latenzzeit von 10 Jahren zwischen der Risikofaktorenexposition und der Krebsinzidenz. Entsprechend wurden Krebsinzidenzdaten aus dem Jahr 2002 und BMIPrävalenzdaten von 1992 für die Kalkulation zugrunde gelegt, was Unterschiede im Vergleich zu den vorliegenden Schätzungen erklären könnte.
Allgemeine Einschränkungen

bei der Interpretation von PAF-Schätzungen

Beim Vergleich von PAF-Schätzungen aus unterschiedlichen Studien muss berücksichtigt werden, dass die Aussagekraft der Schätzungen maßgeblich von den gewählten Referenz- bzw. Expositionskategorien, der verfügbaren Datenqualität für die Expositionsklassifikation, den Risikoschätzern sowie der angewandten Berechnungsmethodik abhängt. Die einzelnen Punkte werden daher im Folgenden näher betrachtet.

Referenzkategorien sollten hinsichtlich der Erreichbarkeit des für die Berechnung zugrunde gelegten Expositionsniveaus differenziert betrachtet werden [6]. Aus diesem Grund wurden neben den Berechnungen mit den Referenzkategorien, wie sie der WCRF verwendet hat, Berechnungen mit alternativen, praxisnäheren Referenzkategorien durchgeführt. Besonders große Unterschiede ergaben sich hier sowohl bei Darm- als auch bei Brustkrebs für die Exposition Alkohol (- Tab.4). Schätzungen mit einer Referenzkategorie von $0 \mathrm{~g} / \mathrm{Tag}$ führten gegenüber einer Referenzkategorie von $<5 \mathrm{~g} /$ Tag zu einer mehr als doppelt so hohen PAF. Da ein Expositionsniveau von $0 \mathrm{~g}$ Alkohol/Tag in der gesamten Bevölkerung in unserem Kulturkreis als kaum erreichbar gilt [20], können die mit einer Referenzkategorie von 0 g/Tag kalkulierten PAF lediglich als theoretisch erzielbares Präventionspotenzial angesehen werden. Mit einer praxisnäheren Referenzkategorie von $<5 \mathrm{~g} /$ Tag dürften demnach nur knapp 50\% des theoretisch errechneten Potenzials auch wirklich erreichbar sein. Ähnliches gilt für den Risikofaktor BMI. Bei Verwendung der Referenzkategorie $<21$ werden beim gegenwärtigen Verteilungsmuster des BMI in der deutschen Bevölkerung deutlich mehr Personen als Risikopersonen klassifiziert als bei einer Referenzkategorie von $<25$. Das so ermittelte präventive Potenzial wird damit künstlich erhöht, da dieses Niveau in der Praxis kaum von der gesamten Bevölkerung erreicht werden kann [20]. Referenzkategorien sind somit immer auch ein Kompromiss aus Praxisrelevanz (z. B. $<5$ g Alkohol/Tag) und theoretisch mini- malem Risiko (z. B. 0 g Alkohol/Tag). Dabei muss auch berücksichtigt werden, dass Expositionen bzw. bestimmte Ausprägungen von Expositionen nicht für alle möglichen Endpunkte gleichermaßen protektiv wirken. Zum Beispiel kann ein BMI $<21$ aus gesundheitlicher Sicht nicht uneingeschränkt empfohlen werden [21]. Berrington de Gonzalez et al. [21] zeigten in ihrer Metaanalyse, dass die Beziehung zwischen BMI und Mortalität J-förmig ist und der „optimale“ BMI zwischen 22,5 und 24,9 liegt. Das Mortalitätsrisiko schwankt jedoch abhängig von Alter und Geschlecht [21, 32]. So steigt der BMI, der mit einem erhöhten Mortalitätsrisiko assoziiert ist, bei Frauen nach dem 50. Lebensjahr auf $>36$, während Adipositas nach dem 65. Lebensjahr kaum noch mit einer verkürzten Lebenserwartung assoziiert ist [32]. In diesem Zusammenhang wäre zu überlegen, bei den Berechnungen zum Präventionspotenzial nach Alter zu stratifizieren und für die verschiedenen Altersgruppen unterschiedliche BMI-Referenzkategorien festzulegen.

Neben der Wahl der Referenzkategorie kann bei den vorliegenden Schätzungen auch die Zusammenfassung mehrerer Expositionskategorien zu einer gemeinsamen Kategorie das PAF-Ergebnis beeinflussen. Beispielsweise wurden in den ausgewählten Kohortenstudien auch BMIKategorien $>30$ erhoben und für diese Risikoschätzer berechnet. Die entsprechenden RR sind zum Teil höher als die ausgewählten RR für die verwendeten Expositionskategorien der vorliegenden Schätzungen. Der Risikogruppe mit einem BMI $>30$ wurde somit ein geringeres Risiko zugeordnet, als ursprünglich in den Kohortenstudien ermittelt, sodass das geschätzte Präventionspotenzial demnach höher liegen könnte. Darüber hinaus besteht für die Klassifizierung von Personen zu verschiedenen Alkoholexpositionskategorien grundsätzlich die Gefahr, dass der Alkoholkonsum in Verzehrserhebungen wie der NVS II unterschätzt wurde (Phänomen des „underreporting“; [8]). Eine Unterschätzung des Alkoholkonsums führt zu einer Fehlklassifikation der Prävalenzen in den Risikoexpositionskategorien, wodurch die Prävalenz in den niedrigeren Expositionskategorien fälschlicherweise ansteigt. Dies führt in der Folge zur 
Unterschätzung der populationsattributablen Fraktion, da ein Teil der Bevölkerung fälschlicherweise einer geringeren Risikoexposition zugeordnet wird.

Als weiterer wichtiger Faktor mit Einfluss auf das Ergebnis der populationsattributablen Fraktion ist der Risikoschätzer zu nennen. Ein höherer Risikoschätzer führt bei gleicher Kategorieeinteilung und gleichen Risikoprävalenzen innerhalb der Expositionskategorien grundsätzlich zu einem höheren PAF-Ergebnis. Auch hier konnten die Vergleichsrechnungen mit Risikoschätzern aus anderen Studien zeigen, dass unterschiedliche relative Risiken zu einer Differenz von 6 bis 7\%-Punkten im Ergebnis führen können. So ergab sich bei der Rechnung für den BMI hinsichtlich Darmkrebses bei Männern je nach gewähltem RR ein Präventionspotenzial von 7 oder 13\% (• Tab.5). Die PAF-Schätzungen differieren auch hier wieder um knapp 50\%, wobei sich die größten Unterschiede bei den Schätzungen zum Alkoholkonsum und Brustkrebs zeigten. Da die Wahl des Risikoschätzers erheblichen Einfluss auf das Ergebnis der populationsattributablen Fraktionen hat, stellt sich die Frage, auf welcher Basis Studien und deren Risikoschätzer für die PAF-Berechnung ausgewählt werden sollten. Vor dem Hintergrund der zunehmenden Bedeutung der Evidenzbasierung sollte zur Ermittlung von PAF-Schätzungen die gegenwärtig bestverfügbare Evidenz genutzt werden, also möglichst Metaanalysen [33]. Da es auch hier zu beträchtlichen Unterschieden in den Risikoschätzern kommen kann, gilt es, bei der Publikation von Schätzungen zum Präventionspotenzial die herangezogenen Studien, Risikoschätzer, verwendeten Referenz- bzw. Expositionskategorien sowie deren Limitationen genau zu beschreiben, um eine angemessene Interpretation und Vergleichbarkeit der Ergebnisse zu ermöglichen.

Schließlich hat auch die Berechnungsmethodik Einfluss auf die PAF-Schätzungen. Die hier angewandte WCRF-Methode ist nur eine Variante von vielen [6]. Alternative Methoden berücksichtigen beispielsweise auch die rechtsschiefe Verteilung des Alkoholkonsums in der Bevölkerung, setzen den Alkoholkonsum als kontinuierliche Variable anstelle von Exposi- tionskategorien ein oder berücksichtigen Latenzzeiten zwischen der Risikofaktorenexposition und dem Auftreten einer Erkrankung [26, 29].

Abschließend muss bei der Interpretation von Schätzungen zum Präventionspotenzial berücksichtigt werden, dass diese auf 2 wesentlichen Modellannahmen beruhen: erstens auf der Kausalität zwischen den betrachteten Risikofaktoren und Krebserkrankungen und zweitens auf der Ceteris-paribus-Bedingung. Die risikoerhöhende Wirkung von Alkohol und Übergewicht für Darm- und Brustkrebs wurde in mehreren systematischen Reviews und Metaanalysen gezeigt [26, 34, 35,36 ] und als ,überzeugend“ klassifiziert $[3,36]$. Darüber hinaus sprechen plausible biologische Wirkmechanismen für eine kanzerogene Wirkung sowohl von Alkohol als auch von übermäßigem Körperfett [3, 36], sodass von einem kausalen Zusammenhang zwischen den Risikofaktoren und den betrachteten Endpunkten ausgegangen werden kann. Allerdings besteht die Möglichkeit, dass es aufgrund von Korrelationen der Risikofaktorenexposition mit nicht adjustierten Risikofaktoren oder anderen bislang unbekannten Faktoren zu einer Unter- (bei einer positiven Korrelation) oder Überschätzung (bei einer negativen Korrelation) der wahren PAF-Schätzungen kommt [29]. Auch die zweite Annahme der Ceteris-paribus-Bedingung ist grundsätzlich mit Unsicherheit verbunden. Diese unterstellt, dass alle weiteren Einflussfaktoren außer der jeweiligen untersuchten Größe (Alkoholkonsum bzw. BMI) als konstant angenommen werden. Sofern bestimmte Entwicklungstrends für andere krebsassoziierte Risikofaktoren vorliegen (z. B. Abnahme der Raucherprävalenz, Veränderungen bei der Verschreibungshäufigkeit von Hormonersatztherapien), sollten auch die Auswirkungen dieser Trends auf die PAFSchätzungen diskutiert werden.

\section{Schlussfolgerungen}

Zusammenfassend lässt sich festhalten, dass die vorgenommenen Schätzungen für Darm- und Brustkrebs in Deutschland ein bedeutsames Präventionspotenzial für Maßnahmen zur Beibehaltung bzw. zum Erreichen einer Normalgewich- tigkeit und zur Reduktion des Alkoholkonsums in der Bevölkerung zeigen. Dabei ist dieses Potenzial geschlechtsspezifisch unterschiedlich hoch. Anhand der Vergleichsrechnungen konnte gezeigt werden, dass die PAF-Schätzungen abhängig vom eingesetzten Risikoschätzer und von den verwendeten Referenzbzw. Expositionskategorien erheblich variieren. Über das tatsächliche Präventionspotenzial geben die hier vorliegenden Daten daher keine genaue Auskunft, da die Schätzungen je nach Auswahl der Parameter um bis zu 50\% schwankten. Aufgrund der dargestellten Unterschiede in der Berechnung von Präventionspotenzialen und den daraus folgenden Abweichungen in den PAF-Ergebnissen müssen vergleichende Interpretationen der Schätzungen dementsprechend vorsichtig und mit Einschränkungen erfolgen. Darüber hinaus sollte bei Vorliegen unterschiedlicher Schätzungen zum Präventionspotenzial gut begründet werden, welche von ihnen als Basis für Entscheidungen zu Präventionsmaßnahmen herangezogen werden. Werden die beschriebenen Einschränkungen bei der Interpretation von populationsattributablen Fraktionen berücksichtigt, können solche Schätzungen zur Beurteilung der Wirksamkeit theoretisch diskutierter Präventionsmaßnahmen nützlich sein und als Grundlage für die Entscheidungsfindung dienen.

\section{Korrespondenzadresse}

\section{A. Wienecke \\ Zentrum für Krebsregisterdaten, Abteilung für Epidemiologie und Gesundheitsberichterstattung, Robert Koch-Institut \\ General-Pape-Str. 62-66, 12101 Berlin \\ WieneckeA@rki.de}

Interessenkonflikt. Der korrespondierende Autor gibt für sich und seine Koautoren an, dass kein Interessenkonflikt besteht.

\section{Literatur}
1. Robert Koch-Institut (2012) Beiträge zur Gesund- heitsberichterstattung des Bundes - Krebs in Deutschland 2007/2008, 8. Ausgabe. Robert Koch- Institut und die Gesellschaft der epidemiologi- schen Krebsregister in Deutschland e.V. (Hrsg) Ber- lin


2. Pritzkuleit R, Beske F, Katalinic A (2010) Demografischer Wandel und Krebserkrankungen in der Zukunft. Onkologie 33(Suppl 7):19-24

3. World Cancer Research Fund, American Institute for Cancer Research (2007) Food, nutrition, physical activity and the prevention of cancer: a global perspective. AICR, Washington

4. World Cancer Research Fund, American Institute for Cancer Research (2009) Policy and action for cancer prevention. Food, nutrition, and physical activity: a global perspective. AICR, Washington

5. Weltgesundheitsorganisation (WHO) (2012) Fact Sheet Cancer Nr. 297. http://www.who.int/mediacentre/factsheets/fs297/en/index.html

6. Rockhill B, Newman B, Weinberg C (1998) Use and misuse of population attributable fractions. Am J Public Health 88(1):15-19

7. Rehm J, Taylor B, Patra J, Gmel G (2006) Avoidable burden of disease: conceptual and methodological issues in substance abuse epidemiology. Int J Methods Psychiatr Res 15(4):181-191

8. Gmel G, Rehm J (2006) Zusammenfassende Gesundheitsmaße von Sterblichkeit und Krankheit: Der steinige Weg zwischen PYLL, YLD, DALY und HALE. Suchttherapie 7:143-153

9. Max Rubner-Institut (Hrsg) (2008) Nationale Verzehrsstudie II, Ergebnisbericht Teil 1, Nationale Verzehrsstudie II, Ergebnisbericht Teil 2

10. Wei EK, Giovannucci E, Wu K et al (2004) Comparison of risk factors for colon and rectal cancer. Int J Cancer 108:433-442

11. Ferrari $P$, Janeb $M$, Norat $T$ et al (2007) Lifetime and baseline alcohol intake and risk of colon and rectal cancers in the European Prospective Investigation into Cancer and Nutrition (EPIC). Int J Cancer 121:2065-2072

12. Engeland A, Tretli S, Austad G, Bjorge T (2005) Height and body mass index in relation to colorectal and gallbladder cancer in two million Norwegian men and women. Cancer Causes Control 16:987-996

13. Pischon T, Lahmann $\mathrm{PH}$, Boeing $\mathrm{H}$ et al (2006) Body size and risk of colon and rectal cancer in the European Prospective Investigation into Cancer and Nutrition (EPIC). J Nat Cancer Inst 98(13):920-931

14. Willett WC, Stampfer MJ, Colditz GA et al (1987) Moderate alcohol consumption and the risk of breast cancer. N Eng J Med 316:1174-1180

15. Tjonneland A, Christensen J, Olsen A et al (2007) Alcohol intake and breast cancer risk: the European Prospective Investigation into Cancer and Nutrition (EPIC). Cancer Causes Control 18:361373

16. Brandt PA van den, Spiegelman D, Yaun SS et al (2000) Pooled analysis of prospective cohort studies on height, weight, and breast cancer risk. Am J Epidemiol 152(6):514-527

17. Lahmann PH, Hoffmann K, Allen N et al (2004) Body size and breast cancer risk: findings from the European Prospective Investigation into Cancer and Nutrition (EPIC). Int J Cancer 111:762-771

18. Levin ML (1953) The occurrence of lung cancer in men. Acta Union Int Contra Cancrum 9:531-541

19. Weiderpass $E$ (2010) Lifestyle and cancer risk. J Prev Med Public Health 43(6):459-471

20. Becker N (2004) Krebsprävention - epidemiologische Aspekte. Onkologe 10:127-138

21. Berrington Gonzalez A, Hartge P, Cerhan JR et al (2010) Body-mass index and mortality among 1.46 million white adults. N Eng J Med 363:2211-2219
22. Europäische Kommission. Bericht zur gesundheitlichen Situation der Frauen in der Europäischen Gemeinschaft. KOM1997/224. http://europa.eu/legislation_summaries/public_health/health_determinants_environment/c11558_de.htm (Zugegriffen: 22.05.2012)

23. Ezzati M, Lopez AD, Rodgers A et al (2002) Selected major risk factors and global and regional burden of disease. Lancet 360:1347-1360

24. Danaei G, Vander Hoorn S, Lopez AD et al (2005) Causes of cancer in the world: comparative risk assessment of nine behavioural and environmental risk factors. Lancet 366:1784-1793

25. Boffetta P, Hashibe M, La Vecchia C et al (2006) The burden of cancer attributable to alcohol drinking. Int J Cancer 119(4):884-887

26. Renehan AG, Soerjomataram I, Tyson M et al (2010) Incident cancer burden attributable to excess body mass index in 30 European countries. Int J Cancer 126:692-702

27. Danaei G, Ding EL, Mozaffarian D et al (2009) The preventable causes of death in the United States: comparative risk assessment of lifestyle, dietary and metabolic risk factors. PLoS Med 6(4):e1000058

28. International Agency for Research on Cancer (IARC) (2007) Attributable causes of cancer in France in the year 2000. IARC Working Group Reports 3. IARC, Lyon

29. Parkin DM, Boyd L, Darby SC et al (2011) The fraction of cancer attributable to lifestyle and environmental factors in the UK in 2010. BJC 105(Suppl 2)

30. Olsen JH, Andersen A, Dreyer L et al (1997) Avoidable cancers in the Nordic countries. APMIS (Suppl 76):1-146

31. Schütze M, Boeing H, Pischon T et al (2011) Alcohol attributable burden of incidence of cancer in eight European countries based on results from prospective cohort study. BMJ 342:d1584

32. Lenz M, Richter T, Mühlhauser I (2009) Morbidität und Mortalität bei Übergewicht und Adipositas im Erwachsenenalter - Eine systematische Übersicht. Dtsch Ärztebl Int 106(40):641-648

33. Renehan AG, Soerjomataram I, Leitzmann MF (2010) Interpreting the epidemiologic evidence linking obesity and cancer: a framework for population-attributable risk estimation in Europe. Eur J Cancer 46:2581-2592

34. Moskal A, Norat T Ferrari P et al (2007) Alcohol intake and colorectal cancer risk: a dose response meta-analysis of published cohort studies. Int J Cancer 120:664-671

35. Hamajima N, Hirose K, Tajima K et al (2002) Alcohol, tobacco and breast cancer: collaborative reanalysis of individual data from 53 epidemiological studies, including 58.515 women with breast cancer and 95.067 women without the disease. BJC 87:1234-1245

36. International Agency for Research on Cancer (2010) Alcohol consumption and ethyl carbamate In: International Agency for Research on Cancer, World Health Organization (Hrsg) IARC monographs on the evaluation of carcinogenic risks to humans, Bd. 96. Lyon

\section{Gesundes Altwerden}

Zum gesunden Altwerden gehören viele unterschiedliche Aspekte, die bisher nur teilweise in ihren Zusammenhängen erkannt worden sind. Bevölkerungsbasierte Längsschnittstudien sind notwendig, um das komplexe Zusammenspiel zwischen körperlichen, psychischen und sozialen Faktoren zum gesunden Altwerden zu beleuchten, mit dem Ziel, die behinderungsfreie Lebenszeit weiter auszudehnen.

Die Ausgabe 04/2012 der Zeitschrift für Gerontologie und Geriatrie gibt in seinem Themenschwerpunkt "Gesundes Altwerden" einen Überblick über aktuelle Studien. Es beinhaltet u.a. folgende Beiträge:

- Ressourcen und Risiken im Alter. Validierung und erste Ergebnisse aus der Longitudinalen Urbanen Cohorten-Alters-Studie (LUCAS)

- Grauzonen von Gesundheit und Handlungsfähigkeit. Erfassung und Aufschlüsselung durch erweiterte Assessments in der LUCAS

- Effekte körperlicher Aktivität und körperlichen Trainings auf den psychischen Status bei älteren Menschen mit und ohne kognitive Schädigung

- Physical activity and other health-related factors predict health care utilisation in older adults. The ActiFE Ulm study

- Gesunderhaltung und Entlastung pflegender Angehöriger von Demenzkranken durch ein „initiales Case Management". Erfahrungen aus dem Ulmer Leuchtturmprojekt Demenz (ULTDEMStudie)

Bestellen Sie diese Ausgabe zum Preis von EUR 34,- zzgl. Versandkosten bei:

Springer Customer Service Center GmbH Kundenservice Zeitschriften

Haberstr. 7 69126 Heidelberg

Tel.: +49 6221-345-4303

Fax: $+496221-345-4229$

leserservice@springer.com

P.S. Vieles mehr rund um $\mathrm{lhr}$ Fachgebiet finden Sie auf www.springermedizin.de 